It is seen that $U$ is identical with the first integral in (3). Thus for a circle, $U$ differs from $F$ by a constant, and so $V$ is the harmonic conjugate of $F$.

For regions not too far from circular it is to be expected that $U+C$ where $C$ is a constant would approximate the solution of the Dirichlet problem. Of course $U+C$ would not take on the correct boundary values. By the maximum principle it results that the greatest error would be on the boundary; hence the error could be determined.

Since the error in the above procedure is harmonic, a second approximation could be set up, etc. This would lead to the method of the arithmetic mean devised by Neumann to solve the Dirichlet problem in convex regions.

\title{
ON SOME EFFECTS OF VELOCITY DISTRIBUTION IN ELECTRON STREAMS
}

Quarterly of Applied Mathematics, XII, 105-116 (1954)

By S. V. YADAVALLI

Although the details of the derivation are different Equations (83), (73) and (67) derived earlier by J. K. Knipp (see: "Klystrons and Microwave Triodes", M.I.T. Radiation Laboratory Series, Vol.7, McGraw-Hill, Chapter 5) are somewhat more general than the corresponding Equations (11), (14) and (43a) of my paper. This was not indicated in my above paper due to an oversight. The author would like to thank Professor J. K. Knipp for kindly drawing his attention to this matter. 